\title{
Partial Hecke-type operators and their applications
}

\author{
Aykut Ahmet Aygunes and Yilmaz Simsek*
}

"Correspondence:

ysimsek63@gmail.com

Department of Mathematics,

Faculty of Science, Akdeniz

University, Campus, Antalya, 07058, Turkey

\begin{abstract}
The aim of this paper is to give not only the matrix representation of partial Hecke-type operators by means of Bernoulli polynomials and Euler polynomials, but also functional equations and differential equations related to partial Hecke-type operators and special polynomials. By using these functional equations and differential equations, we derive some identities associated with special polynomials and partial Hecke-type operators. Moreover, we find several useful identities and relations using the partial Hecke operators.
\end{abstract}

MSC: 05B20; 11B68; $11 \mathrm{~F} 25$

Keywords: Bernoulli polynomial; Euler polynomial; partial Hecke-type operator; total Hecke-type operator; Hurwitz zeta function; partial zeta function

\section{Introduction}

Recently, there have been many applications of Bernoulli polynomials and Euler polynomials in differential equations, in analytic number theory and in engineering. High-order linear differential-difference equations have also been solved in terms of Bernoulli polynomials. These polynomials are also related to several linear operators. In this paper, we investigate and derive several new identities related to the Hecke-type operators and generating functions for special polynomials.

Recently, many authors introduced and investigated the following generating functions which give us the Bernoulli polynomials $B_{n}(x)$ and the Euler polynomials $E_{n}(x)$, respectively:

$$
\frac{t e^{x t}}{e^{t}-1}=\sum_{n=0}^{\infty} B_{n}(x) \frac{t^{n}}{n !} \quad(|t|<2 \pi)
$$

and

$$
\frac{2 e^{x t}}{e^{t}+1}=\sum_{n=0}^{\infty} E_{n}(x) \frac{t^{n}}{n !} \quad(|t|<\pi)
$$

For $x=0$, (1) and (2) are reduced to the generating functions for the Bernoulli numbers $B_{n}$ and the Euler numbers $E_{n}$, respectively $(c f .[1-4])$, and see also the references cited in each of these earlier works.

@ 2013 Aygunes and Simsek; licensee Springer. This is an Open Access article distributed under the terms of the Creative Commons Attribution License (http://creativecommons.org/licenses/by/2.0), which permits unrestricted use, distribution, and reproduction in any medium, provided the original work is properly cited. 
The multiplication formulas for the Bernoulli and Euler polynomials are given as follows:

$$
\sum_{k=0}^{m-1} B_{n}\left(\frac{x+k}{m}\right)=m^{1-n} B_{n}(x)
$$

and for odd $m$,

$$
\sum_{k=0}^{m-1}(-1)^{k} E_{n}\left(\frac{x+k}{m}\right)=m^{1-n} E_{n}(x)
$$

(cf. [5-8]), and see also the references cited in each of these earlier works.

The Bernoulli polynomials satisfy the following well-known identity:

$$
\frac{1}{m} \sum_{k=0}^{m-1} f\left(x+\frac{k}{m}\right)=m^{-n} f(m x)
$$

where $m$ and $n$ are positive integers $(c f .[5,9,10])$.

Bayad et al. [11] introduced and systematically studied the following family of partial Hecke-type operators on $\mathbb{C}[x]$.

Throughout this paper, we use the following notations: $a \equiv 1(N)$. Let $\mathbb{N}=\{0,1,2,3, \ldots\}$ and $\mathbb{Z}^{+}=\{1,2,3, \ldots\}$.

For fixed $a, N \in \mathbb{Z}^{+}$and $0 \leq k \leq a-1$, we have

$$
T_{\chi_{a, N}}(P(x))=\sum_{k=0}^{a-1} \chi_{a, N}(k) P\left(\frac{x+k}{a}\right)
$$

where

$$
\chi_{a, N}(k)= \begin{cases}\xi_{N}^{k}=e^{\frac{2 \pi i k}{N}} & \text { if } N \geq 2, \\ \frac{1}{a} & \text { if } N=1 .\end{cases}
$$

Lemma 1.1 [11, p.114, Lemma 1] For any $a, N \in \mathbb{Z}^{+}$such that $a \equiv 1(N)$, we have the following properties:

(i) $T_{\chi_{a, N}}$ preserves the degree in $\mathbb{C}[x]$.

(ii) By induction,

$$
T_{\chi_{a, N}}\left(x^{m}\right)= \begin{cases}S_{0}=1 & \text { if } m=0 \\
a^{-m} x^{m}+a^{-m} \sum_{v=0}^{m-1}\left(\begin{array}{c}
m \\
v
\end{array}\right) S_{m-v}\left(\chi_{a, N}\right) x^{v} & \text { if } m \geq 1\end{cases}
$$

where

$$
S_{m-v}\left(\chi_{a, N}\right)=\sum_{k=0}^{a-1} \chi_{a, N}(k) k^{m-v}
$$

(iii) For any $m \in \mathbb{Z}^{+}$, let $\beta_{m}=\left(1, x, x^{2}, \ldots, x^{m}\right)$ be the canonical $\mathbb{C}$-basis of

$$
\mathbb{C}_{m}[x]=\{P(x) \in \mathbb{C}[x]: \operatorname{deg} P(x) \leq m\}
$$


Then the matrix $M_{\beta_{m}}\left(T_{\chi_{a, N}}\right)$ corresponding to the operator $T_{\chi_{a, N}}$ (restricted to $\left.\mathbb{C}_{m}[x]\right)$ in the basis $\beta_{m}$ is represented by:

$$
M_{\beta_{m}}\left(T_{\chi_{a, N}}\right)=\left(\begin{array}{ccccc}
S_{0}\left(\chi_{a, N}\right) & a^{-1} S_{1}\left(\chi_{a, N}\right) & a^{-2} S_{2}\left(\chi_{a, N}\right) & \cdots & a^{-m} S_{m}\left(\chi_{a, N}\right) \\
0 & a^{-1} S_{0}\left(\chi_{a, N}\right) & 2 a^{-2} S_{1}\left(\chi_{a, N}\right) & \cdots & a^{-m}\left(\begin{array}{c}
m \\
1
\end{array}\right) S_{m-1}\left(\chi_{a, N}\right) \\
0 & 0 & a^{-2} S_{0}\left(\chi_{a, N}\right) & \cdots & a^{-m}\left(\begin{array}{c}
m \\
2
\end{array}\right) S_{m-2}\left(\chi_{a, N}\right) \\
0 & 0 & 0 & \cdots & a^{-m}\left(\begin{array}{c}
m \\
3
\end{array}\right) S_{m-3}\left(\chi_{a, N}\right) \\
\vdots & \vdots & \vdots & & \vdots \\
0 & 0 & 0 & \cdots & a^{-m} S_{0}\left(\chi_{a, N}\right)
\end{array}\right)
$$

for all $0 \leq l \leq m-1$.

(iv) Let $a, b \geq 1$ such that $a \equiv b \equiv 1(N)$, then

$$
T_{\chi a, N} T_{\chi b, N}=T_{\chi b, N} T_{\chi a, N} .
$$

Consequently, for a given integer $n$, there is only one monic polynomial $P_{n, N}$ with degree $n$ in $x$ satisfying the functional equation (6).

The operator $T_{\chi a, N}$ satisfies the following equation:

$$
T_{\chi a, N}\left(P_{n, N}(x)\right)=a^{-n} P_{n, N}(x)
$$

For $a \equiv 1(N)$, from (6) and Lemma 1.1, we know that $P_{n, N}(x)$ is a monic polynomial ( $c f$. [11]).

Remark 1.2 Equations (3) and (4) are closely related to the functional equation of (6). For $N=1$ and $N=2$, equation (6) is reduced to $P_{n, 1}(x)=B_{n}(x)$ and $P_{n, 2}(x)=E_{n}(x)$, respectively. For fixed $a, N \in \mathbb{Z}^{+}$, we know that there is only one monic polynomial satisfying (6) by Lemma 1.1, and there already exist the functional equations as (3) and (4).

The total Hecke-type operators, associated with partial Hecke-type operators, are defined by Bayad et al. [11, p.112, Eq. (1.6)] as follows:

$$
T_{N}=\sum_{a \equiv 1(N)} T_{\chi a, N} .
$$

Theorem 1.3 [11] Polynomials $P_{n, N}(x)$ are eigenfunctions for the operators $T_{N}$ with eigenvalues $N^{-n} \zeta\left(n, \frac{1}{N}\right)$, that is,

$$
T_{N}\left(P_{n, N}(x)\right)=N^{-n} \zeta\left(n, \frac{1}{N}\right) P_{n, N}(x)
$$

where $\zeta(s, x)$ is the Hurwitz zeta function defined by

$$
\zeta(s, x)=\sum_{k \geq 0} \frac{1}{(x+k)^{s}} \quad(c f .[10,12])
$$




\section{Differential equations related to the partial Hecke-type operators and special polynomials}

In this section, we derive some ordinary and partial differential equations not only for a generating function, but also for partial Hecke-type operators. We also give a functional equation for the generating function. We set

$$
F_{N}(t, x)=\sum_{n=0}^{\infty} P_{n, N}(x) \frac{t^{n}}{n !}
$$

We now give an explicit formula of the generating function $F_{N}(t, x)$ as follows.

Theorem 2.1 [11] Generating functions for the polynomials $P_{n, N}(x)$ are given by

$$
F_{N}(t, x)= \begin{cases}\frac{t e^{t x}}{e^{t}-1} & \text { if } N=1 \\ \frac{\left(\xi_{N}-1\right) e^{t x}}{\xi_{N} e^{t}-1} & \text { if } N \geq 2\end{cases}
$$

The polynomials $P_{n, N}(x)$ are the so-called Bernoulli-Euler-type polynomials.

We derive the following partial differential equation for $F_{N}(t, x)$ as follows:

$$
\frac{\partial^{v}}{\partial x^{v}} F_{N}(t, x)=t^{v} F_{N}(t, x)
$$

Theorem 2.2 Let $v \in \mathbb{N}$. Then

$$
\frac{d^{v}}{d x^{v}} P_{n, N}(x)= \begin{cases}(n)_{v} B_{n-v}(x) & \text { if } N=1 \\ (n)_{v} P_{n-v, N}(x) & \text { if } N \geq 2\end{cases}
$$

where $(n)_{v}=n(n-1)(n-2) \cdots(n-v+1)$.

Proof By using (7), for $N \geq 2$, we obtain

$$
\sum_{n=0}^{\infty}\left(\frac{d^{v}}{d x^{v}} P_{n, N}(x)\right) \frac{t^{n}}{n !}=\sum_{n=v}^{\infty}(n)_{v} P_{n-v, N}(x) \frac{t^{n}}{n !}
$$

Therefore, by comparing the coefficients of $\frac{t^{n}}{n !}$ on both sides of equation (8), we have the desired result.

For $N=1$, we apply the same process. So, we omit it.

We set the following differential equation:

$$
\frac{\xi_{N} e^{t(x+y)}}{\left(\xi_{N} e^{t}-1\right)^{2}}=(x+y-1) \frac{e^{t(x+y-1)}}{\left(\xi_{N} e^{t}-1\right)}-\frac{d}{d t} \frac{e^{t(x+y-1)}}{\left(\xi_{N} e^{t}-1\right)} .
$$

\section{Theorem 2.3}

$$
\frac{\xi_{N}}{\xi_{N}-1} \sum_{k=0}^{n}\left(\begin{array}{l}
n \\
k
\end{array}\right) P_{k, N}(x) P_{n-k, N}(y)=(x+y-1) P_{n, N}(x+y-1)-n P_{n+1, N}(x+y-1) .
$$


Proof We make some arrangement (9) and obtain

$$
\begin{aligned}
\frac{\xi_{N}}{\left(\xi_{N}-1\right)^{2}}\left[\left(\frac{\xi_{N}-1}{\xi_{N} e^{t}-1}\right)^{2} e^{t(x+y)}\right]= & \frac{x+y-1}{\xi_{N}-1}\left(\frac{\left(\xi_{N}-1\right) e^{t(x+y-1)}}{\left(\xi_{N} e^{t}-1\right)}\right) \\
& -\frac{d}{d t} \frac{1}{\xi_{N}-1} \frac{\left(\xi_{N}-1\right) e^{t(x+y-1)}}{\left(\xi_{N} e^{t}-1\right)} .
\end{aligned}
$$

Therefore,

$$
\frac{\xi_{N}}{\left(\xi_{N}-1\right)^{2}}\left(\frac{\xi_{N}-1}{\xi_{N} e^{t}-1} e^{t x}\right)\left(\frac{\xi_{N}-1}{\xi_{N} e^{t}-1} e^{t y}\right)=\frac{\xi_{N}}{\left(\xi_{N}-1\right)^{2}}\left(\sum_{n=0}^{\infty} P_{n, N}(x) \frac{t^{n}}{n !}\right)\left(\sum_{n=0}^{\infty} P_{n, N}(y) \frac{t^{n}}{n !}\right) .
$$

From the above equation, we get

$$
\begin{aligned}
& \frac{\xi_{N}}{\left(\xi_{N}-1\right)^{2}} \sum_{n=0}^{\infty} \sum_{k=0}^{n}\left(\left(\begin{array}{l}
n \\
k
\end{array}\right) P_{k, N}(x) P_{n-k, N}(y)\right) \frac{t^{n}}{n !} \\
& =\frac{x+y-1}{\xi_{N}-1} \sum_{n=0}^{\infty}\left(P_{n, N}(x+y-1)-\frac{n}{\xi_{N}-1} P_{n+1, N}(x+y-1)\right) \frac{t^{n}}{n !} .
\end{aligned}
$$

By comparing the coefficients of $\frac{t^{n}}{n !}$ on both sides of the above equation, we have the desired result.

Remark 2.4 In Theorem 2.3, we obtain a convolution formula for the polynomials $P_{n, N}(x)$. If we substitute $x=y=1$ into Theorem 2.3, then we get a convolution formula for the Eulerian-type numbers $(c f .[10,13])$.

Higher-order partial differential equation for $T_{\chi_{a, N}}\left(P_{n, N}(x)\right)$ is given by the following theorem.

Theorem 2.5 Let $N \geq 2$ and $v \in \mathbb{N}$. Then

$$
\frac{\partial^{v}}{\partial x^{v}} T_{\chi a, N}\left(P_{n, N}(x)\right)=\frac{(n)_{v}}{a^{v}} T_{\chi a, N}\left(P_{n-v, N}(x)\right),
$$

where

$$
(n)_{v}=n(n-1)(n-2) \cdots(n-v+1) .
$$

Proof Taking $v$ th derivative of the operator $T_{\chi_{a, N}}\left(P_{n, N}(x)\right)$, with respect to $x$, we obtain the following higher-order partial differential equation:

$$
\frac{\partial^{v}}{\partial x^{v}} T_{\chi_{a, N}}\left(P_{n, N}(x)\right)=\sum_{k=0}^{a-1} \chi_{a, N}(k) \frac{\partial^{v}}{\partial x^{v}} P_{n, N}\left(\frac{x+k}{a}\right) .
$$

Using Theorem 2.2, we get

$$
\frac{\partial^{v}}{\partial x^{v}} T_{\chi_{a, N}}\left(P_{n, N}(x)\right)=\frac{(n)_{v}}{a^{v}} \sum_{k=0}^{a-1} \chi_{a, N}(k) P_{n-v, N}\left(\frac{x+k}{a}\right) .
$$

Thus, we get the desired result. 


\section{Matrix representations of partial Hecke-type operators}

In this section, we give some numerical examples for the matrix representations of the operator $T_{\chi_{a, N}}$. For the basis $\beta_{m}=\left\{1, x, x^{2}, \ldots, x^{m}\right\}$, our matrix representations contain Bernoulli polynomials and Euler polynomials for the operators $T_{\chi_{a, 1}}$ and $T_{\chi_{a, 2}}$, respectively. Therefore, we need the following lemmas.

Lemma 3.1 Let $m, n \in \mathbb{N}$ and $n \geq 1$. Then

$$
\sum_{k=0}^{n-1} k^{m}=\frac{B_{m+1}(n)-B_{m+1}(0)}{m+1} .
$$

Lemma 3.2 Let $m, n \in \mathbb{N}$ and $n \geq 1$. Then

$$
\sum_{k=0}^{n-1}(-1)^{k} k^{m}=\frac{E_{m}-(-1)^{n} E_{m}(n)}{2} .
$$

Proofs of Lemma 3.1 and Lemma 3.2 have been given by many authors (among others) (cf. $[2,4,8,10])$.

In a special case, substituting $N=1$ into (iii) in Lemma 1.1 and using Lemma 3.1, we get

$$
\begin{aligned}
S_{l, 1}(a) & =\sum_{k=0}^{a-1} \chi_{a, 1}(k) k^{l} \\
& =\frac{B_{l+1}(a)-B_{l+1}(0)}{a(l+1)} .
\end{aligned}
$$

According to the above equation, we are ready to give the main result of this section by the following theorem.

Theorem 3.3 The matrix $M_{\beta_{m}}\left(T_{\chi_{a, 1}}\right)$ corresponding to the operator $T_{\chi_{a, 1}}$ (restricted to $\left.\mathbb{C}_{m}[x]\right)$ in the basis $\beta_{m}$ is represented by Bernoulli polynomials as follows:

$$
M_{\beta_{m}}\left(T_{\chi_{a, 1}}\right)=\left(\begin{array}{ccccc}
\frac{B_{1}(a)-B_{1}(0)}{a} & \frac{B_{2}(a)-B_{2}(0)}{2 a^{2}} & \frac{B_{3}(a)-B_{3}(0)}{3 a^{3}} & \cdots & \frac{B_{m+1}(a)-B_{m+1}(0)}{a^{m+1}(m+1)} \\
0 & \frac{B_{1}(a)-B_{1}(0)}{a^{2}} & \frac{B_{2}(a)-B_{2}(0)}{a^{3}} & \cdots & \left(\begin{array}{c}
m \\
1
\end{array}\right) \frac{B_{m}(a)-B_{m}(0)}{a^{m+1} m} \\
0 & 0 & \frac{B_{1}(a)-B_{1}(0)}{a^{3}} & \cdots & \left(\begin{array}{c}
m \\
2
\end{array}\right) \frac{B_{m-1}(a)-B_{m-1}(0)}{a^{m+1}(m-1)} \\
0 & 0 & 0 & \cdots & \left(\begin{array}{c}
m \\
3
\end{array}\right) \frac{B_{m-2}(a)-B_{m-2}(0)}{a^{m+1}(m-2)} \\
\vdots & \vdots & \vdots & & \vdots \\
0 & 0 & 0 & \cdots & \frac{B_{1}(a)-B_{1}(0)}{a^{m+1}}
\end{array}\right) .
$$

Setting $N=2$ (iii) in Lemma 1.1 and using Lemma 3.2, we obtain

$$
\begin{aligned}
S_{l, 2}(a) & =\sum_{k=0}^{a-1} \chi_{a, 2}(k) k^{l} \\
& =\frac{E_{l}(0)-(-1)^{a} E_{l}(a)}{2} .
\end{aligned}
$$

If $a \equiv 1(2)$, then we obtain another main result by the following theorem. 
Theorem 3.4 Let a be an odd number. The matrix $M_{\beta_{m}}\left(T_{\chi a, 2}\right)$ corresponding to the operator $T_{\chi a, 2}$ (restricted to $\mathbb{C}_{m}[x]$ ) in the basis $\beta_{m}$ is represented by Euler polynomials as follows:

$$
M_{\beta_{m}}\left(T_{\chi_{a, 2}}\right)=\left(\begin{array}{ccccc}
\frac{E_{0}(0)+E_{0}(a)}{2} & \frac{E_{1}(0)+E_{1}(a)}{2 a} & \frac{E_{2}(0)+E_{2}(a)}{2 a^{2}} & \cdots & \frac{E_{m}(0)+E_{m}(a)}{2 a^{m}} \\
0 & \frac{E_{0}(0)+E_{0}(a)}{2 a} & \frac{E_{1}(0)+E_{1}(a)}{a^{2}} & \cdots & \left(\begin{array}{c}
m \\
1
\end{array}\right) \frac{E_{m-1}(0)+E_{m-1}(a)}{2 a^{m}} \\
0 & 0 & \frac{E_{0}(0)+E_{0}(a)}{2 a^{2}} & \cdots & \left(\begin{array}{c}
m \\
2
\end{array}\right) \frac{E_{m-2}(0)+E_{m-2}(a)}{2 a^{m}} \\
0 & 0 & 0 & \cdots & \left(\begin{array}{c}
m \\
3
\end{array}\right) \frac{E_{m-3}(0)+E_{m-3}(a)}{2 a^{m}} \\
\vdots & \vdots & \vdots & & \vdots \\
0 & 0 & 0 & \cdots & \frac{E_{0}(0)+E_{0}(a)}{2 a^{m}}
\end{array}\right) .
$$

\section{Some applications of total Hecke-type operators}

In this section, we give some applications related to eigenvalues for the total Hecke-type operators of $T_{1}$ and $T_{2}$. We derive many new identities which are related not only to the total Hecke-type operators, but also to the Riemann zeta function, the Hurwitz zeta function, Bernoulli and Euler numbers, Euler identities and the convolution of Bernoulli and Euler numbers and polynomials.

Throughout this section, we use the following notation:

$$
\zeta^{\prime}(a)=\left.\frac{d}{d s} \zeta(s)\right|_{s=a}
$$

The partial zeta function $H(s, a, F)$ is defined by

$$
H(s, a, F)=\sum_{n \equiv a(F)} \frac{1}{n^{s}}
$$

where $\Re(s)>1, n>0$ and $0<a<F\left(F \in \mathbb{Z}^{+}\right)(c f .[4,8,10,12,14])$.

Theorem 4.1 The polynomials $P_{n, N}(x)$ are eigenfunctions for the operators $T_{N}$ with eigenvalues $H(n, 1, N)$, that is,

$$
T_{N}\left(P_{n, N}(x)\right)=H(n, 1, N) P_{n, N}(x),
$$

where $H(s, a, F)$ is a partial zeta function.

Proof

$$
\sum_{n \equiv a(F)} \frac{1}{n^{s}}=\frac{1}{F^{s}} \zeta\left(s, \frac{a}{F}\right)
$$

Therefore,

$$
\zeta\left(s, \frac{a}{F}\right)=F^{s} H(s, a, F) .
$$

Substituting $F=N, s=n$ and $a=1$ into the above equation, after using Theorem 1.3, we arrive at the desired result. 
Theorem 4.2 Let $n \in \mathbb{Z}^{+}$with $n>1$. Then we have

$$
T_{2}\left(E_{2 n}(x)\right)=\frac{(-1)^{n}\left(1-2^{-2 n}\right)(2 \pi)^{2 n}}{(4 n+2)(2 n) !} E_{2 n}(x) \sum_{k=1}^{n-1}\left(\begin{array}{c}
2 n \\
2 k
\end{array}\right) B_{2 k} B_{2 n-2 k} .
$$

Proof Putting $N=2$ in Theorem 1.3 and using

$$
P_{n, 2}(x)=E_{n}(x),
$$

we have

$$
T_{2}\left(E_{n}(x)\right)=2^{-n} \zeta\left(n, \frac{1}{2}\right) E_{n}(x) .
$$

We recall from the definition of $\zeta\left(n, \frac{1}{2}\right)$ and $\zeta(n)$ that we have

$$
2^{-n} \zeta\left(n, \frac{1}{2}\right)=\left(1-2^{-n}\right) \zeta(n)
$$

(cf. [10, p.96]). Combining (11) and (12), we get

$$
T_{2}\left(E_{n}(x)\right)=\zeta(n)\left(1-2^{-n}\right) E_{n}(x) .
$$

If we replace $n$ by $2 n$ in the above equation, we obtain

$$
T_{2}\left(E_{2 n}(x)\right)=\zeta(2 n)\left(1-2^{-2 n}\right) E_{2 n}(x) .
$$

From the work of Srivastava and Choi [4, p.98], we recall that

$$
\zeta(2 n)=\frac{2}{2 n+1} \sum_{k=1}^{n-1} \zeta(2 k) \zeta(2 n-2 k)
$$

where $n \in \mathbb{Z}^{+}$with $n>1$ and

$$
\zeta(2 n)=\frac{(-1)^{n+1}(2 \pi)^{2 n} B_{2 n}}{2(2 n) !} .
$$

By substituting (15) and (16) into (14), after some elementary calculations, we arrive at the desired result.

Theorem 4.3 Let $n \in \mathbb{N}$. Then

$$
T_{2}\left(E_{2 n}(x)\right)=\frac{(-1)^{n+1}\left(2^{2 n}-1\right) \pi^{2 n}}{2(2 n) !} B_{2 n} E_{2 n}(x) .
$$

Proof Combining (14) and (16), we easily complete the proof of the theorem, that is,

$$
T_{2}\left(E_{2 n}(x)\right)=\zeta(2 n)\left(1-2^{-2 n}\right) E_{2 n}(x)=\frac{(-1)^{n+1}\left(2^{2 n}-1\right) \pi^{2 n}}{2(2 n) !} B_{2 n} E_{2 n}(x) .
$$

By using (10) and (17), we obtain a convolution formula (Euler identity) for Bernoulli numbers. 
Theorem 4.4 Let $n>1$. Then

$$
B_{2 n}=-\frac{1}{2 n+1} \sum_{k=1}^{n-1}\left(\begin{array}{l}
2 n \\
2 k
\end{array}\right) B_{2 k} B_{2 n-2 k}
$$

Proof Since the left-hand sides of (10) and (17) are equal, the right-hand sides of (10) and (17) must be equal. Thus, we obtain

$$
\frac{(-1)^{n+1}\left(2^{2 n}-1\right) \pi^{2 n}}{2(2 n) !} B_{2 n}=\frac{(-1)^{n}\left(2^{2 n}-1\right) \pi^{2 n}}{(4 n+2)(2 n) !} \sum_{k=1}^{n-1}\left(\begin{array}{l}
2 n \\
2 k
\end{array}\right) B_{2 k} B_{2 n-2 k} .
$$

After some elementary calculation in the above equation, we get the desired result.

Observe that the proof of (18) is also given in [4].

Theorem 4.5 Let $n \in \mathbb{N}$. Then

$$
T_{2}\left(E_{2 n}(x)\right)=\frac{e^{\pi i n} \pi^{2 n}}{4(2 n-1) !} E_{2 n-1}(0) E_{2 n}(x) .
$$

Proof For all $n \in \mathbb{N}$, we have

$$
E_{2 n-1}(0)=\frac{4(-1)^{n}}{(2 \pi)^{2 n}}(2 n-1) !\left(2^{2 n}-1\right) \zeta(2 n)
$$

(cf. [4, p.131]). By using (14) and (19), we obtain

$$
\begin{aligned}
T_{2}\left(E_{2 n}(x)\right) & =\zeta(2 n)\left(1-2^{-2 n}\right) E_{2 n}(x) \\
& =\left(\frac{(2 \pi)^{2 n} E_{2 n-1}(0)}{4(-1)^{n}(2 n-1) !\left(2^{2 n}-1\right)}\right)\left(1-2^{-2 n}\right) E_{2 n}(x) .
\end{aligned}
$$

Thus, the proof is completed.

Theorem 4.6 Let $n \in \mathbb{N}$. Then we have

$$
\begin{aligned}
& T_{2}\left(E_{2 n+1}(x)\right) \\
& \quad=\frac{(-1)^{n+1}\left(1-2^{-2 n-1}\right)(2 \pi)^{2 n+1} E_{2 n+1}(x)}{2(2 n+1) !} \int_{0}^{1} B_{2 n+1}(t) \cot (\pi t) d t .
\end{aligned}
$$

Proof Consider that $n$ is replaced by $2 n+1$ in (13), we have

$$
T_{2}\left(E_{2 n+1}(x)\right)=\zeta(2 n+1)\left(1-2^{-2 n-1}\right) E_{2 n+1}(x) .
$$

For all $n \in \mathbb{N}$, one can easily get

$$
\zeta(2 n+1)=\frac{(-1)^{n+1}(2 \pi)^{2 n+1}}{2(2 n+1) !} \int_{0}^{1} B_{2 n+1}(t) \cot (\pi t) d t
$$


(cf. [4, p.99, Eq. (21)]). Hence, we have

$$
\begin{aligned}
T_{2}\left(E_{2 n+1}(x)\right) & =\zeta(2 n+1)\left(1-2^{-2 n-1}\right) E_{2 n+1}(x) \\
& =\left(\frac{(-1)^{n+1}\left(1-2^{-2 n-1}\right)(2 \pi)^{2 n+1}}{2(2 n+1) !} \int_{0}^{1} B_{2 n+1}(t) \cot (\pi t) d t\right) E_{2 n+1}(x) .
\end{aligned}
$$

Thus, the proof is completed.

Theorem 4.7 Let $n \in \mathbb{N}$. Then we have

$$
T_{2}\left(E_{2 n+1}(x)\right)=\frac{2(-1)^{n}(2 \pi)^{2 n}\left(1-2^{-2 n-1}\right)}{(2 n) !} \zeta^{\prime}(-2 n) E_{2 n+1}(x) .
$$

Proof Note that, for all $n \in \mathbb{N}$, we have

$$
\zeta^{\prime}(-2 n)=(-1)^{n} \frac{(2 n) !}{2(2 \pi)^{2 n}} \zeta(2 n+1)
$$

(cf. [4, p.99, Eq. (22)]). By using (21) and (24), we have

$$
\begin{aligned}
T_{2}\left(E_{2 n+1}(x)\right) & =\zeta(2 n+1)\left(1-2^{-2 n-1}\right) E_{2 n+1}(x) \\
& =\left(\frac{2(-1)^{n} \zeta^{\prime}(-2 n)(2 \pi)^{2 n}}{(2 n) !}\right)\left(1-2^{-2 n-1}\right) E_{2 n+1}(x) .
\end{aligned}
$$

Thus, the proof is completed.

Theorem 4.8 Let $n \in \mathbb{N}$. Then

$$
T_{1}\left(B_{2 n}(x)\right)=\frac{(-1)^{n+1} 2^{2 n-1} \pi^{2 n}}{(2 n) !} B_{2 n} B_{2 n}(x) .
$$

Proof Substituting $N=1$ into Theorem 1.3 and by $P_{n, 1}(x)=B_{n}(x)$, we have

$$
T_{1}\left(B_{n}(x)\right)=\zeta(n, 1) B_{n}(x)=\zeta(n) B_{n}(x)
$$

If $n$ is replaced by $2 n$ in the above equation, we get

$$
T_{1}\left(B_{2 n}(x)\right)=\zeta(2 n) B_{2 n}(x)
$$

By using (16), we have

$$
T_{1}\left(B_{2 n}(x)\right)=\frac{(-1)^{n+1}(2 \pi)^{2 n} B_{2 n}}{2(2 n) !} B_{2 n}(x) .
$$

Thus, the proof is completed.

Theorem 4.9 Let $n \in \mathbb{Z}^{+}$with $n>1$. Then we have

$$
T_{1}\left(B_{2 n}(x)\right)=\frac{(-1)^{n}(2 \pi)^{2 n}}{(4 n+2)(2 n) !} B_{2 n}(x) \sum_{k=1}^{n-1}\left(\begin{array}{l}
2 n \\
2 k
\end{array}\right) B_{2 k} B_{2 n-2 k} .
$$


Proof By using (26), (15) and (16), we have

$$
\begin{aligned}
T_{1}\left(B_{2 n}(x)\right) & =\zeta(2 n) B_{2 n}(x) \\
& =\left(\frac{2}{2 n+1} \sum_{k=1}^{n-1} \zeta(2 k) \zeta(2 n-2 k)\right) B_{2 n}(x) \\
& =\frac{2}{2 n+1}\left(\sum_{k=1}^{n-1} \frac{(-1)^{k+1}(2 \pi)^{2 k} B_{2 k}}{2(2 k) !} \frac{(-1)^{n-k+1}(2 \pi)^{2 n-2 k} B_{2 n-2 k}}{2(2 n-2 k) !}\right) B_{2 n}(x) \\
& =\frac{(-1)^{n}(2 \pi)^{2 n} B_{2 n}(x)}{(4 n+2)(2 n) !} \sum_{k=1}^{n-1}\left(\begin{array}{c}
2 n \\
2 k
\end{array}\right) B_{2 k} B_{2 n-2 k .}
\end{aligned}
$$

Thus, the proof is completed.

Theorem 4.10 Let $n \in \mathbb{N}$. Then

$$
T_{1}\left(B_{2 n}(x)\right)=\frac{(-1)^{n}(2 \pi)^{2 n}}{4(2 n-1) !\left(2^{2 n}-1\right)} E_{2 n-1}(0) B_{2 n}(x) .
$$

Proof By using (26) and (19), we have

$$
\begin{aligned}
T_{1}\left(B_{2 n}(x)\right) & =\zeta(2 n) B_{2 n}(x) \\
& =\left(\frac{(2 \pi)^{2 n} E_{2 n-1}(0)}{4(-1)^{n}(2 n-1) !\left(2^{2 n}-1\right)}\right) B_{2 n}(x) .
\end{aligned}
$$

Thus, the proof is completed.

Theorem 4.11 Let $n \in \mathbb{N}$. Then

$$
T_{1}\left(B_{2 n+1}(x)\right)=\frac{(-1)^{n+1}(2 \pi)^{2 n+1}}{2(2 n+1) !} B_{2 n+1}(x) \int_{0}^{1} B_{2 n+1}(t) \cot (\pi t) d t .
$$

Proof By replacing $n$ by $2 n+1$ in (25), we have

$$
T_{1}\left(B_{2 n+1}(x)\right)=\zeta(2 n+1) B_{2 n+1}(x) .
$$

By substituting (29) into (22), we get

$$
T_{1}\left(B_{2 n+1}(x)\right)=\frac{(-1)^{n+1}(2 \pi)^{2 n+1}}{2(2 n+1) !} B_{2 n+1}(x) \int_{0}^{1} B_{2 n+1}(t) \cot (\pi t) d t .
$$

Thus, the proof is completed.

Theorem 4.12 Let $n \in \mathbb{N}$. Then we have

$$
T_{1}\left(B_{2 n+1}(x)\right)=\frac{2(-1)^{n}(2 \pi)^{2 n}}{(2 n) !} \zeta^{\prime}(-2 n) B_{2 n+1}(x) .
$$


Proof By using (29) and (24), we have

$$
\begin{aligned}
T_{1}\left(B_{2 n+1}(x)\right) & =\zeta(2 n+1) B_{2 n+1}(x) \\
& =\left(\frac{2(-1)^{n} \zeta^{\prime}(-2 n)(2 \pi)^{2 n}}{(2 n) !}\right) B_{2 n+1}(x) .
\end{aligned}
$$

Thus, the proof is completed.

By comparing (20) and (23) or (28) and (30), we arrive at the following result.

\section{Corollary 4.13}

$$
\int_{0}^{1} B_{2 n+1}(t) \cot (\pi t) d t=-\frac{2(2 n+1)}{\pi} \zeta^{\prime}(-2 n) .
$$

\section{Competing interests}

The authors declare that they have no competing interests.

\section{Authors' contributions}

All authors completed the paper together. All authors read and approved the final manuscript.

\section{Acknowledgements}

Dedicated to Professor Hari M Srivastava.

All authors are partially supported by Research Project Offices Akdeniz Universities. We would like to thank the referees for their valuable comments.

\section{Received: 14 November 2012 Accepted: 22 January 2013 Published: 6 March 2013}

\section{References}

1. Ozden, H, Simsek, Y, Srivastava, HM: A unified presentation of the generating functions of the generalized Bernoulli, Euler and Genocchi polynomials. Comput. Math. Appl. 60, 2779-2787 (2010)

2. Kim, T, Rim, S-H, Simsek, Y, Kim, D: On the analogs of Bernoulli and Euler numbers, related identities and zeta and L-functions. J. Korean Math. Soc. 45, 435-453 (2008)

3. Simsek, Y: Twisted $(h, q)$-Bernoulli numbers and polynomials related to twisted $(h, q)$-zeta function and $L$-function J. Math. Anal. Appl. 324, 790-804 (2006)

4. Srivastava, HM, Choi, J: Series Associated with the Zeta and Related Functions. Kluwer Academic, Dordrecht (2001)

5. Carlitz, L: A note on the multiplication formulas for the Bernoulli and Euler polynomials. Proc. Am. Math. Soc. 4, 184-188 (1953)

6. Luo, Q-M, Srivastava, HM: Some generalizations of the Apostol-Bernoulli and Apostol-Euler polynomials. J. Math. Anal. Appl. 308, 290-302 (2005)

7. Raabe, J: Zurückführung einiger summen and bestimmten integrale auf die Jacob Bernoullische function. J. Reine Angew. Math. 42, 348-376 (1851)

8. Srivastava, HM, Kim, T, Simsek, Y: q-Bernoulli numbers and polynomials associated with multiple $q$-zeta functions and basic L-series. Russ. J. Math. Phys. 12, 241-268 (2005)

9. Lehmer, DH: A new approach to Bernoulli polynomials. Am. Math. Mon. 95, 905-911 (1998)

10. Srivastava, HM, Choi, J: Zeta and $q$-Zeta Functions and Associated Series and Integrals. Elsevier, Amsterdam (2012)

11. Bayad, A, Aygunes, AA, Simsek, Y: Hecke operators and generalized Bernoulli-Euler polynomials. J. Algebra Number Theory, Adv. Appl. 3, 111-122 (2010)

12. Whittaker, ET, Watson, GN: A Course of Modern Analysis, 4th edn. Cambridge University Press, Cambridge (1962)

13. Chu, W, Zhou, RR: Convolutions of Bernoulli and Euler polynomials. Sarajevo J. Math. 6, 147-163 (2010)

14. Washington, LC: Introduction to Cyclotomic Fields. Springer, Berlin (1982) 